This article was originally published in:

G. Meyer, J. Valldorf (Ed.), Advanced Microsystems for Automotive Applications 2009, Springer-Verlag

Berlin, pp. 187-208, 2009

It is reproduced here with minor typographical corrections.

\title{
Evaluation of ADAS with a supported-driver model for desired allocation of tasks between human and technology performance
}

A.P. van den Beukel, M.C. van der Voort, University of Twente, Laboratory Design, Production and Management, the Netherlands

\begin{abstract}
Partly automated driving is relevant for solving mobility problems, but also causes concerns with respect to the driver's reliability in task performance. The supported driver model presented in this paper is therefore intended to answer the question, what type of support and in which circumstances, will enhance the driver's ability to control the vehicle. It became apparent that prerequisites for performing tasks differ per driving task's type and require different support. The possible support for each driving task's type, has been combined with support-types to reduce the error causations from each different performance level (i.e. knowledge-based, rule-based and skill-based performance). The allocation of support in relation to performance level and driving task's type resulted in a supported driver model and this model relates the requested circumstances to appropriate support types. Among three tested ADAS systems, semiautomated parking showed best allocation of support; converting the demanding parallel parking task into a rather routine-like operation.
\end{abstract}

\section{Introduction}

The expectations of using technical solutions for solving mobility problems are very high. The reasoning behind the expected advantages is generally that assistance systems are, in comparison to human drivers, superior with respect to precision of operation and ability to operate under severe circumstances. Being more precise and having faster reaction times, automated cars are presumed to cause less accidents and therefore reduce congestion [1]. Demonstrations with prototype vehicles - for example: Darpa Urban Challenge - show already the technical capabilities for completely automated driving. Due to reasons of practicality, liability and user preferences [2] automation of the complete driving task is, however, not likely to become reality in the mid-term future. For this reason there will exist transitions between human (driver) operation and system (vehicle) operation when specific driving tasks are automated.

Human Factors experts argue that partly automated driving also has drawbacks. If a driving task is supported by an assistance system, this could cause for example mental underload and loss of skills, resulting in a decrease in driver reliability [3]. Endsley \& Kiris [4] provided insight that automation of a task can reduce situation awareness by placing the operator out-of-the-loop and gave evidence that improving single aspects of the driving task (like reaction time) does not necessarily mean that the driving task as a whole improves. However, several studies show positive effects of automated driving on road efficiency [5]. In view of both the relevance that automated driving has for solving mobility problems, and the expressed concerns, it is important to answer the question: In what driving circumstances is automation beneficial? Therefore this paper presents a supported-driver model which helps to specify these circumstances and relate them to appropriate support types. Therewith this model will assist the development of appropriate applications for partly automated driving.

Starting point for the development of the model are existing models for human task performance and the pursuit that the overall goal of assistance should be to enhance the ability of the joint driver-vehicle system to remain in control throughout the travel [6]. Three Advanced Driver Assistance Systems (ADAS) will be evaluated to what respect they are in line with the supported-driver model. The results will also be used to appraise the supported driver model as a possible means for further developing driver assistance, like facilitating the transitions between driver and vehicle operation for partly automated driving. 


\section{Models of the driving tasks}

To compile a model for desired allocation of support among driving tasks, this section captures existing models for human task performance. It is important to acknowledge first, that the purposes for which these models have been generated differ. Some models are intended to evaluate (new) ADAS with respect to driving safety, other models reflect typical driving behaviour to be applied within traffic simulations in order to assess traffic efficiency. Consequently, some models are more normative and others rather descriptive. As the aspired model is intended to help the development of driver interfaces which might not yet be existing, our model will be descriptive too.

\subsection{Driving as series of control loops}

Several models exist which describe driving tasks as series of control loops, combining input with feedforward and feedback control. Within these models specific names are defined for the steps within a control loop. McRuer [7], for example, considers driving as a sequence of operations, characterised by: "visual search", then "decisionmaking" and subsequently "effecting the desired actuation". Other authors use different names, but in fact these models all consider driving as a set of control loops, being performed within one or several sequences of input, throughput and output. Dirken [8] uses the same distinction in his Human-machine-interface (HMI) model to explain how human and machine cooperate and interact to execute a task. The HMI-model defines a sequence of input, throughput and output both at the human and machine-side that will be cycled through to achieve a task, possibly during multiple phases. This model is very useful as it considers both human and machine. See figure 1 for a representation of the model. Within this model, human input considers perception of information. This information is applied within the step throughput to evaluate and make decisions. The result of these decisions, human's reactions, are represented as output. Human output is then considered to be input for a machine. It contains information which will be processed within the machine by means of an input, throughput and output phase as well.

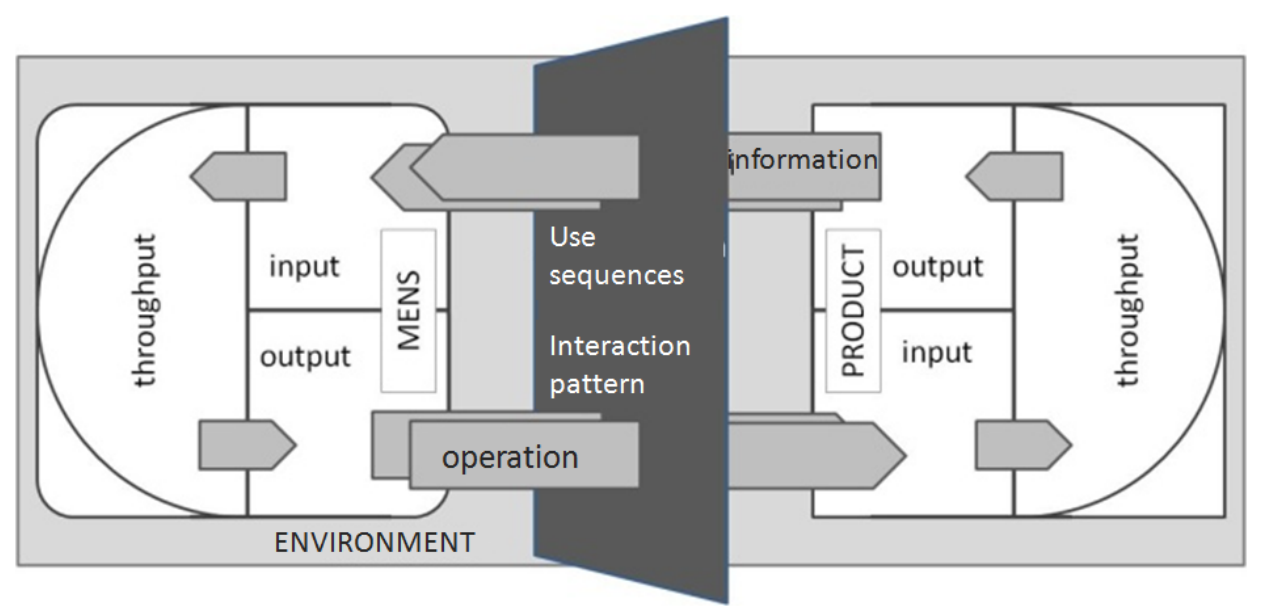

Fig. 1. Human-Machine-Interface model (Dirken, 1997) visualizes task performance as a series of control loops.

\subsection{Rasmussen's model for task performance}

Rasmussen [9] makes a general distinction for performing tasks, based on the degree of experience and distinguishes three levels: the knowledge-based level, the rule-based level and the skill-based level. Between these levels Rasmussen also differentiates whether the control-loop is being phased through in a closed-loop or in an open-loop fashion. At the highest knowledge-based level, human behaviour is goal-controlled and depends upon feedbackcorrection. Knowledge-based tasks are therefore performed within a closed-loop process. This is the level at which people develop new ways of problem solving. It requires attention and effort. Steps involved in this control loop are demanding, especially the decision making. At the rule-based level, tasks are involved that are more familiar. The results of (previous) actions are available and have become like rules to the person that is performing. Once a rule is chosen the actions are carried out in a rather automatically fashion. The control process is mostly a closed-loop one, but the steps within this loop are less demanding. At the lowest skill-based level, highly practiced routines are carried out. Actions are completely automated. The control process is an open-loop one, i.e. there is no continuous feedback mechanism. Only when something goes wrong in this open-loop mode, this will trigger task performance to be carried out at a higher level. With this distinction in levels, Rasmussen considers the amount of mental effort needed for executing a task and therewith addresses a dependency on individual differences in task performance. 


\subsection{Driving task as a hierarchical structure of control levels}

Michon [10] proposed that the driving task could be structured at three levels. This model resulted in a fundamental hierarchy of the different levels within the driving task as a whole. These levels are: the strategic, tactical and operational level. At the strategic level drivers prepare their journey; this concerns general trip planning, route, transportation mode, time, etc. At the tactical level drivers exercise manoeuvring control, allowing to negotiate the directly prevailing traffic circumstances, like crossing an intersection, obstacle avoidance, etc. Here drivers are mostly concerned with interacting with other traffic and the road system. The operational level involves the elementary tasks that have to be performed to be able to manoeuvre the vehicle, mostly performed automatically and unconsciously (like: steering, using pedals or changing gears). Executing a tactical or operational task always facilitates achieving the goals of a task on a superordinated level. Although this model is intended to be an objective descriptive model, the allocation of subtasks might differ per superordinated driving task. For example; regulating speed is considered an operational task in most general circumstances. However, being a subtask of reverse parking, regulating speed might be considered a tactical task.

Michon's hierarchical structure of driving tasks relates to the general HMI-model for executing a task, because each of the three levels within Michon's model can be explained whilst using the HMI-model. The differences between driving task levels can be derived from the duration and amount of recurrences in which the HMI-model is being 'walked through'. The input-throughput-output-loop for a strategic task usually is of long duration; because evaluation if a strategic task has succeeded (e.g. if a goal has been achieved) can only be made upon arrival. Within a strategic task loop, several tactical or operational task loops can be performed. Michon's hierarchical structure of the driving task and Dirken's HMI-model are therefore valuable in helping to explain how a driving task is being executed. Nonetheless, these models do not directly supply insight in desired task allocation for the investigated ADAS. A next step is therefore to relate Michon's descriptive model of the driving task's hierarchy to the general model for task performance from Rasmussen.

\subsection{Relation between driving task's hierarchy and performance level}

The relation between the driving tasks' hierarchy and the general model for task performance from Rasmussen is visualised in figure 2 .

\begin{tabular}{|c|c|c|c|c|}
\hline & & Hierar & hy of driving tasks & (Michon) \\
\hline & & strategic tasks & tactical tasks & operational tasks \\
\hline 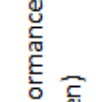 & knowledge-based & \begin{tabular}{|l} 
navigating in \\
unfamiliar area
\end{tabular} & $\begin{array}{l}\text { controlling skidding } \\
\text { car }\end{array}$ & novice on first lesson \\
\hline 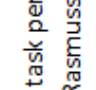 & rule-based & $\begin{array}{l}\text { choice between } \\
\text { familiar routes }\end{array}$ & passing other vehicles & $\begin{array}{l}\text { driving unfamiliar } \\
\text { vehicle }\end{array}$ \\
\hline 足 & skill-based & $\begin{array}{l}\text { route used for daily } \\
\text { commuting }\end{array}$ & $\begin{array}{l}\text { dealing with familiar } \\
\text { intersections }\end{array}$ & $\begin{array}{l}\text { vehicle handling in } \\
\text { familiar circumstances }\end{array}$ \\
\hline
\end{tabular}

Fig. 2. Relation between driving task’s hierarchy and performance level. Adopted from Hale et al. [12].

In general, each type of driving task from the hierarchical ladder can be performed at either knowledge-based, rulebased or skill-based level. The examples in figure 2 show that the performance level which a driver uses to execute a task, is dependent on driving experience and contextual knowledge. Experienced drivers for example could need knowledge-based performance when passing an unfamiliar crossing. For experienced drivers most driving tasks cluster in the three cells on the diagonal from upper-left to lower right. Knowledge-based behaviour is involved at the strategic level, rule-based behaviour at the tactical level and skill-based behaviour at the operational level. As the examples show, exceptions reflect differences between skilled and novice performance, and between familiar and unfamiliar situations. Therefore, it becomes clear that task allocation can only be applied in relation to driving experience and contextual knowledge.

Causes and probability of errors, differ per performance levels [11]. As skill-based tasks are comprised of very familiar actions, which humans perform almost automatically, inattention is the main cause of errors for tasks performed at this level. Rule-based tasks are familiar to the performer. Upon correct recognition of a situation or condition, the performer can apply a stored rule to steer towards a known end goal. Subsequently, the denominating error cause is misinterpretation. Successful performance of the knowledge-based task depends heavily upon the performer's fundamental knowledge, diagnosis, and analysis skills. Unlike rule-based tasks, the performer is not per se able to head for an end goal. In general, drivers operate more homogeneously and predictable at skill-based and rule-based levels than at knowledge-based level. The probability for an error to occur is therefore lower for tasks 
performed at these levels. Altogether, it is recommendable that a support system supports driving tasks to be performed at rule-based or skill-based level rather than at knowledge-based level [12]. This is important insight in order to specify those subtasks for which support is most appropriate.

\section{Support levels}

To develop the required supported driver model, we now focus on possible support levels. Six different levels can be distinguished and are indicated in figure 3. Ranging from passively supporting drivers, by assisting, to actively supporting drivers, by taking over tasks, these levels are: Augmenting, Advising, Warning, Mitigation, Intervention and Automation. The differences between these support levels can be explained by identifying what steps within the HMI-model are being supported. These steps can be any of the input, throughput or output steps at either the human's or machine's side. The first level, augmenting, supports the human's input by emphasizing relevant and important information. For instance offering a camera view with highlighted pedestrians during the night. Advising and warning especially supports the driver's throughput in decision making and evaluation of input. It might also support in drawing attention to specific input. Advises might be given upon and without human's request. Warnings are independent from driver's request. The fourth level, mitigation, aims at tempering criticality of a dangerous situation, for example: pretensioning brakes when the vehicle approaches an obstacle with high relative velocity. Mitigation is a support level that only comes into operation when the driver gives the corresponding output, e.g. braking. Automation and intervention are both support levels in which the machine takes over human's part in the control loop for a specific (sub)task. The difference is that intervention does take over suddenly, in critical situations without prior request, whereas automation is intended to be activated voluntarily for a period of time. Often intervention is intended as safety enhancement, whereas automation is rather regarded to increase comfort.

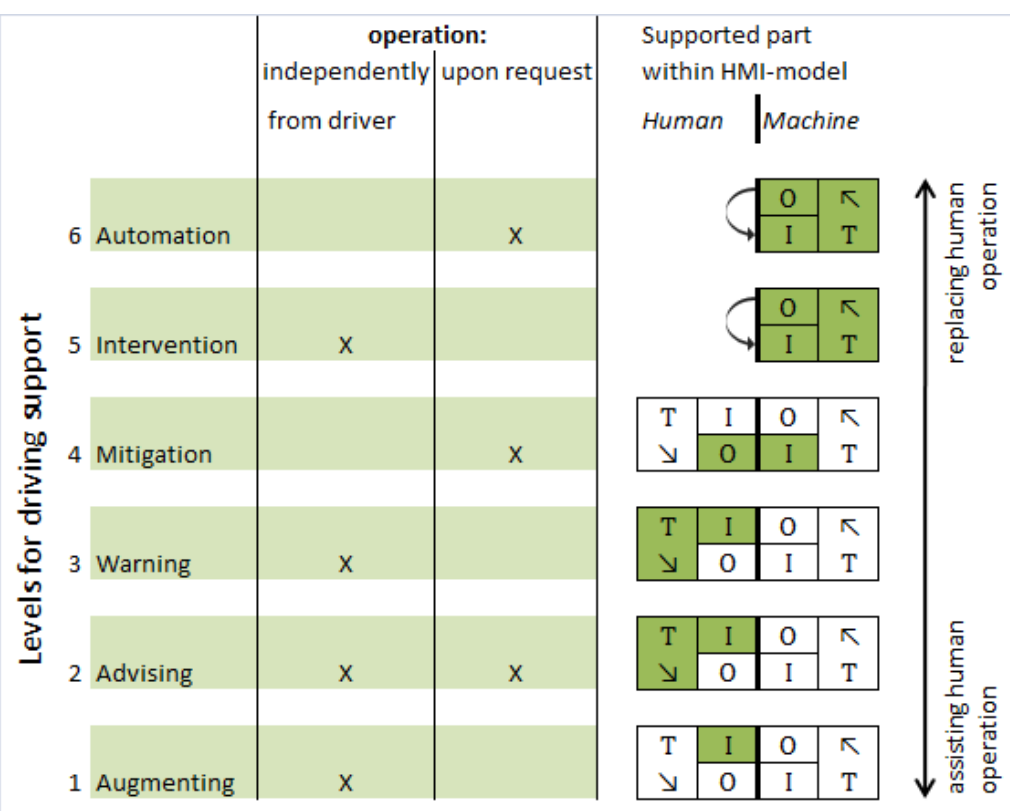

Supported part within HMI-model. I=Input, T=Throughput, $0=$ Output

Fig. 3. Classification of support levels

Figure 3 visualizes the differences between support levels by identifying what step within the HMI-model is being supported. The supported steps are either the input, throughput or output step at the human's side, or the control loop is shortened by automating the human's steps within a subtask.

Related to the classification of support levels is the division of responsibility. Although it is difficult to define a precise separation in responsibilities, the fourth level, mitigation, is considered to mark a change from support levels (i.e. levels 1, 2 and 3) within which the human remains responsible for executing a (sub)task and those support levels whereat the machine is responsible for executing the involved (sub)task. For the support levels automation and intervention, it is clear that the machine is fully responsible as the human is placed out of the control loop. When support of a (sub)task takes place only within the human's input or throughput step, as applies to augmenting, advising and warning, the output remains unsupported and the driver can theoretically operate without being affected by the previous supported control steps. Though assisted by a means of support, the driver is considered to remain responsible for tasks applied with those support types. (Nonetheless, everyone will understand that advises 
and warnings are intended to positively influence human behaviour. Therefore developers have the responsibility to provide correct information. Hence, false warnings or wrong advises might cause deteriorated driving behaviour). Mitigation is a support level with divided responsibility. The machine's control loop is being supported to raise its effectiveness, this will however only be put into effect when the related human output is performed.

A driving task can be composed of several subtasks and when support is applied, the support type might differ per subtasks. Subsequently, responsibility for performing a driving task can be divided over the driver and the machine per subtask. The overall goal of automotive design should be to enhance the ability of the joint driver-vehicle system to remain in control throughout the travel [6]. The identification of responsibility per support type is therefore aimed at ensuring that the allocation of support among the subtasks enables this joint driver-vehicle system to remain in control as good as possible. The allocation of support to subtasks under the condition that responsibility within a subtask is non-ambiguously be either at the driver or at the machine, seems therefore a plausible approach.

\section{Introducing a supported-driver model}

In paragraph 2.4 the relationship between the driving tasks' hierarchy and performance levels has been explained and was visualized in a driving task vs. performance matrix. It became apparent that the level of performance for executing a type of driving task depends on the level of (driving) experience and the contextual knowledge (i.e. familiarity with the location). To continue developing the required supported-driver model, we now implement the preferred support types within the matrix.

\subsection{A supported-driver model dependent on driving tasks' type and performance level}

The allocation of support among performance-level and driving task type compasses two aspects: The contribution to (1) avoidance of errors and (2) to appropriate prerequisites for performing the driving tasks. As explained in section 2.4, the causes and probability of errors differ per performance level a task is being executed with. For tasks performed at skill-based level, inattention is for example the main cause of error. With respect to the different driving task types, it has been distinguished what the main prerequisites are for proper execution of those tasks. Support types have therefore also been selected based upon their contribution in gaining these prerequisites. Now, the support conditions per performance level and per driving task type have been combined with one another, by selecting what support type fits both conditions. The resulting scheme is visualized in figure 4 .

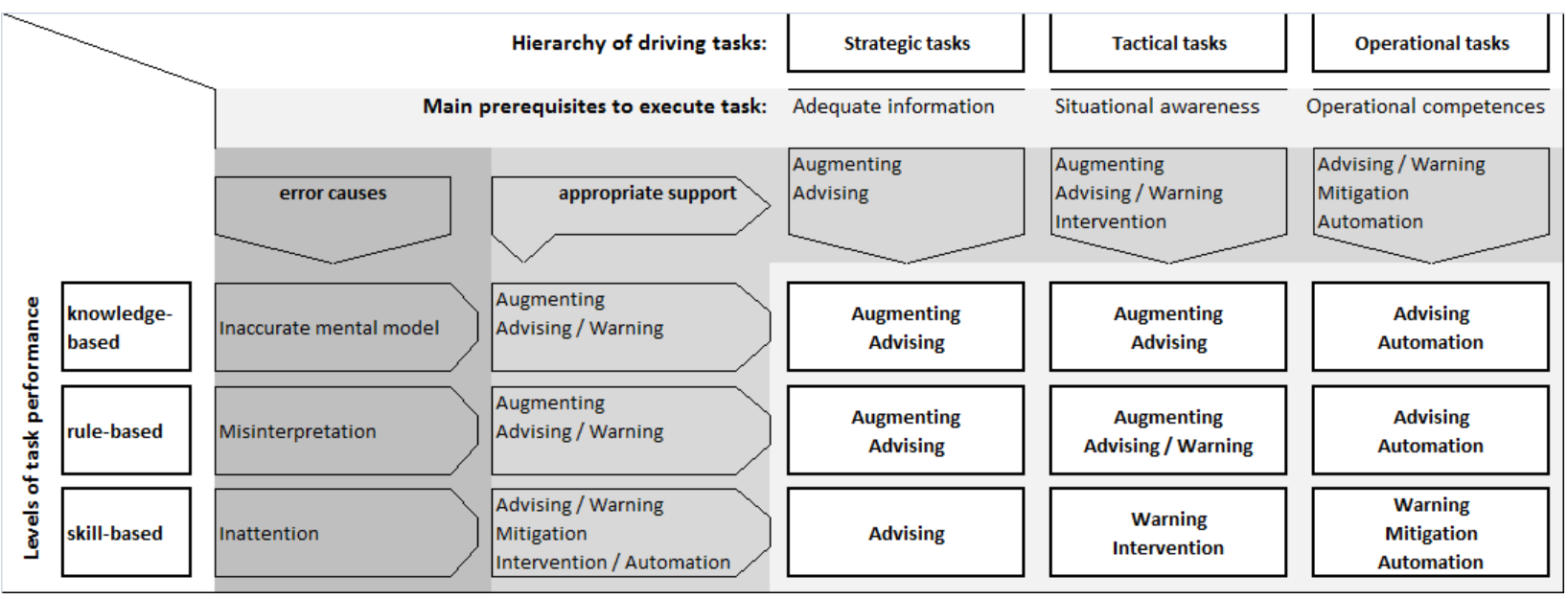

Fig. 4. Preferred support types dependent on driving task and performance level.

As the strategic tasks involve preparation of a journey, adequate information is an important prerequisite to perform these tasks. In general, augmenting sensoric input and advising with regard to the interpretation of this input are adequate support types. However, the error causation on skill-based level is inattention, therefore the preferred support for a strategic task performed at this level is only advising. At the tactical level drivers are mostly concerned with interacting with other traffic and the road system. Compared to the other driving task types, the consequences from errors made at this level are most dangerous. Intervention is therefore an appropriate support to avoid errors caused by inattention. Also warning generally applies to tactical tasks. For tactical tasks performed at knowledge- or rule-based level, misinterpretation is the dominating cause of error, therefore advising or warning is appropriate for this task as well. Perception and interpretation are especially important to gain good situational awareness. Augmenting is therefore a support type that applies especially to tactical tasks, performed at knowledge- and rule- 
based level. Advising and warning are similar types of assistance, both offering informational support. The difference is whether this information is requested or not. The same difference is with automation and intervention: Automation is voluntarily, but intervention is not. Besides, intervention is normally intended for a short instance. The consideration whether this support should be voluntarily or not is dependent on the situation. If an operational task is operated at knowledge-based level for example, the support type advising is especially useful to assist learning and to develop appropriate competences.

Automation of an operational task performed at knowledge-based level avoids mistakes made by novice drivers due to lack of knowledge and competences. Therewith this support type can be an important benefit for road safety. Hence, automation of operational tasks reduces the need to develop operational competences. Consequently, this takes away the risk that the drivers make operational mistakes. However, it also takes away the ability for the driver to develop these competences. Therefore automation of an operational task should only be applied if it is ensured that this support will be executed under all circumstances.

\subsection{Advantages of the supported-driver model}

In comparison to existing models for the driving task, the supported driver model helps to specify circumstances for adequate support. These circumstances are differentiated with regard to type of driving task and performance level. This allows to evaluate whether support types applied to existing assistance systems enhance the ability of the joint driver-vehicle system to remain in control under relevant circumstances. Moreover, the enhancement can be compared with the related unassisted driving task as well. Since unassisted subtasks can be placed within the driving task vs. performance matrix as well, these can be evaluated with regard to their relation to error occurrence and error severity. Likewise, section 6 will evaluate three existing ADAS systems. Moreover, the model can be used to examine existing driving tasks and to explore what kind of new support is most adequate for which subtask. Therewith the model can be used for developing future applications for assisted driving, intended to help reducing mobility problems.

\subsection{Considerations when applying the assisted driver model}

The following aspects are important to consider when applying the model:

A driving task consists of one or more subordinated tasks, called subtasks;

With respect to the performance levels of the involved driving tasks, it is desirable that support enables driving tasks to be as much as possible being executed on rule-based and skill-based level, because drivers then behave more homogeneously and predictable;

- As performance levels are dependent on driving experience, the model needs to be verified for a chosen group with defined experience;

The considerations to allocate support types, as explained in the first paragraph of this section, gives reason for the following general recommendations for the development of ADAS:

Enable single subtasks to be performed at preferably skill-based or rule-based level;

When possible reduce the amount of subtasks the human is involved in, but avoid that subtasks are being replaced by subtasks which require a higher performance level;

Automation of operational tasks should be applied to reveal the driver and therewith easing the driver in executing tactical tasks;

Within a single subtask, avoid support types without a clear separation of responsibility between driver and technology;

Preferably, automation of a subtask should be ensured under all applicable circumstances, because loss of skills only then remains without harm;

Besides, the transitions between human and machine operation involved in nonpermanently automated subtasks most often relate to the existence of system boundaries. These system boundaries are often reached in critical circumstances. Therefore applying non-permanently automated subtasks is potentially dangerous; 
When support is temporary, provide clarity with regard to the moment of termination of a supported subtask. Provide also clarity upon who is responsible for terminating the support.

\section{Advanced Driver Assistance Systems}

This section introduces three assistance systems, which will be evaluated with the assisted driver model in the next section. The evaluation focuses on the appropriateness of the applied support to enhance the ability to remain in control within the related driving circumstances. The evaluated systems are intended to support reverse parallel parking, cruising and changing lanes and are described in the next paragraphs.

\subsection{Semi-Automated Parallel Parking (SAPP)}

With this assistance system the vehicle parks in parallel parking slots semi-automatically. One of the first suppliers who developed this system and introduced it on the market is Valeo.

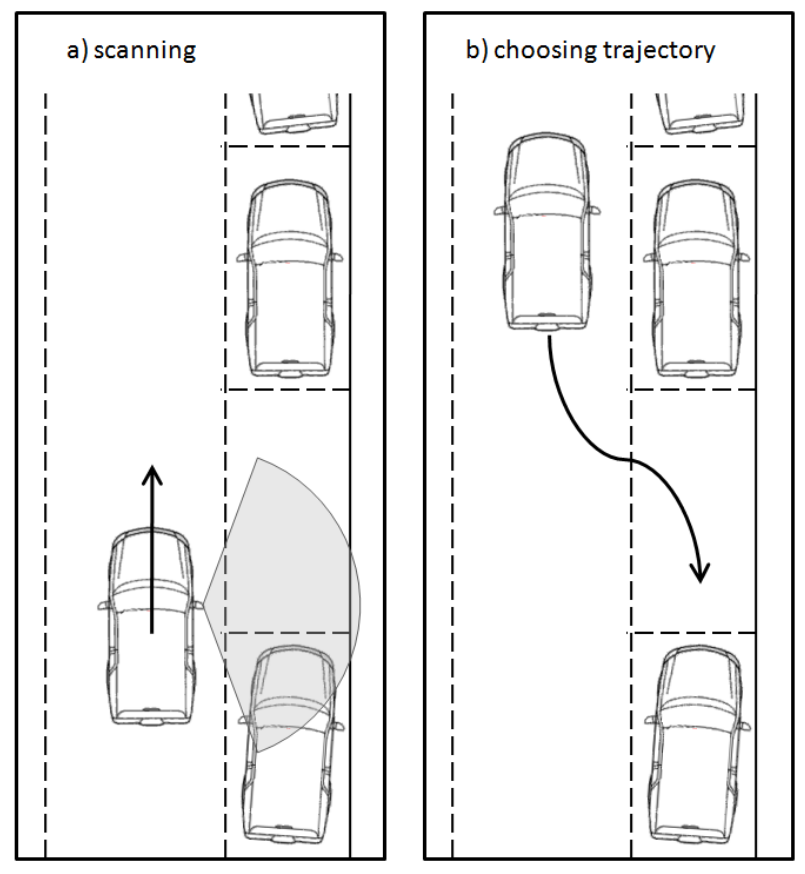

Fig. 5. Process steps for using SAPP.

The Valeo-system is taken as a basis for this case. The system functions as follows, see figure 5. After activation, the system scans for available parking places. For scanning and assessment of the possibilities, the driver needs to pass the complete parking slot. A display indicates when a parking place has sufficient size. As soon as the driver selects reverse driving, the vehicle steers automatically and obtains the correct path for reverse parking. During this reverse parking manoeuvre the driver operates backing up-speed with the gas pedal, brake and probably clutch. Therewith the driver remains responsible for the moment and speed of backing up and can make intermediate stops of the manoeuvre when traffic circumstances require to do so. The most important technologies this system consists of are: parking place measurement, based on radar or a camera; electrically powered steering, trajectory calculation and rear vision.

\subsection{Adaptive Cruise Control (ACC)}

Cruise control is a system to remain speed automatically. Adaptive Cruise Control (ACC) systems use either a radar or laser setup to monitor vehicles in front of the host vehicle and use this information to slow down when approaching another vehicle and to accelerate again to the preset speed if traffic allows to do so. With other words: ACC automates remaining speed and keeping distance. The driver preselects a desired speed; this is displayed in the instrument cluster. Furthermore, the driver chooses between three predefined headway settings. These settings 
involve fixed time based distance stages, therefore the distance to the vehicle in front changes with speed. For the first commercially available ACC systems, the following system boundaries are typically [13]:

ACC operates only from $30 \mathrm{~km} / \mathrm{h}$ onwards;

ACC cannot be used in stop-and-go traffic. When approaching stationary traffic, the driver must take control of the vehicle by braking in good time;

The time gap to a vehicle ahead is usually not less than $1 \mathrm{~s}$. When driving straight ahead, the response of the ACC to a vehicle cutting in close may be delayed. The vehicle that cuts in is not detected before it is positioned on the same lane as the ACC vehicle. As a result the time gap can be below $1 \mathrm{~s}$ for a brief period;

- The system has limited deceleration capabilities. A typically maximum deceleration of $2,0 \mathrm{~m} / \mathrm{s}^{2}$ is provided. Large speed differences, e.g. quickly approaching a heavy goods vehicle, can therefore not be regulated;

Detection of the leading vehicle may be lost when cornering due to the system's limited side field of vision. When cornering, the ACC vehicle is not accelerated to the requested speed for an instance in order not to drive up too close to the leading vehicle.

When the system has reached its functional limits, the driver is requested to assume control by releasing a warning signal. Furthermore, ACC is marketed as a convenience system and driver interventions always have higher priority than the ACC control. Because of automating de- and accelerating, ACC is widely regarded as a key component of any future generations of smart cars.

\subsection{Lane Change Assist (LCA)}

The Lane Change Assist system is intended to support changing lanes by warning if a vehicle is within the driver's blind spot area or if an approaching vehicle does not allow a safe lane change. See figure 6; From driver A's perspective, vehicle $\mathrm{C}$ is within the blind spot area and vehicle $\mathrm{B}$ approaches with a higher relative velocity.

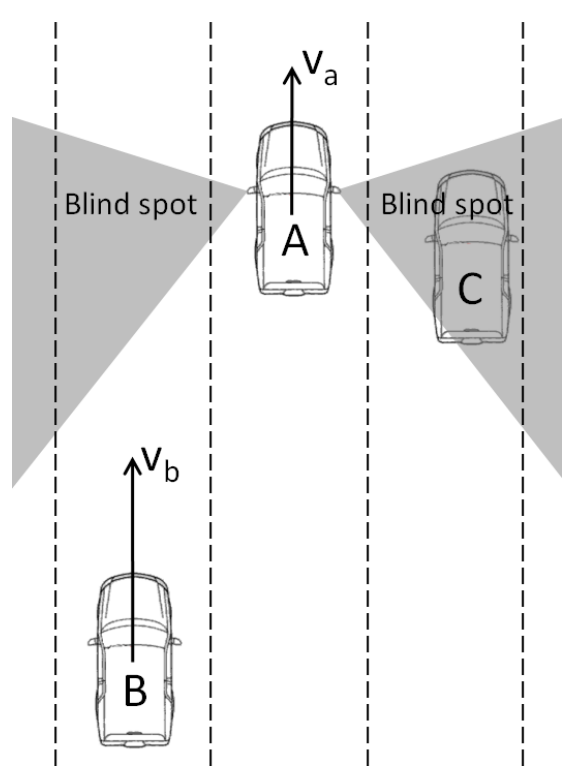

Fig. 6. Critical factors involved in changing lanes

Monitoring the vehicle's blind spot area is especially a problem for lorries and delivery vans. Hence, freight vehicles are available with Blind Spot Detection (BSD). Monitoring relative velocity of approaching vehicles from the rear has been introduced to the market by MobilEye and this system is taken as a basis for this case study [14]. The system uses camera-technology to measure relative speed. The system does not support overtaking within situations with oncoming traffic. The main functions are: (a) tracking of dynamic targets moving towards the vehicle, (b) detection of obstacles at close range, (c) detection of vehicles in the blind spot and (d) detection of critical and noncritical objects in the lateral and rear areas. Apart from monitoring the host vehicle's blind spot area, it also detects approaching vehicles and motorcycles from a distance of $60 \mathrm{~m}$. This data is geared towards indicating to the driver 
whether it is safe to change lane or not. With MobilEye's system the visible alert whether or not it is safe to change lanes, is continuously available at the side mirrors.

\section{Evaluation of ADAS with supported driver model}

\subsection{Assessment of SAPP with respect to preferred support types}

As explained before, SAPP supports the task of reverse parallel parking. To assess this assistance function with respect to the preferred support types, it is important to review first how a driver in general executes reverse parallel parking. This is schematically visualized in the task-level matrix, see figure 7 . The task is being executed in five subtasks. First a parking spot has to be defined which is likely to have the appropriate size (1). This selection is a strategic task, executed at knowledge-based level. Next, the appropriate trajectory needs to be defined to manoeuvre the vehicle along (2). Then, the operation needs to be appropriately timed to avoid hindrance of other road users (3). This is a tactical task, performed at rule-based level. In addition to defining the trajectory, speed needs to be regulated to move the vehicle along the trajectory (4). Finally, the appropriate trajectory needs to be evaluated and possibly be refined (5). When the vehicle is being parked between obstacles -which is often a reason for reverse parallel parking- defining and refining the trajectory (steering the wheel) is a delicate task requiring a sequence of closed-loop control cycles.

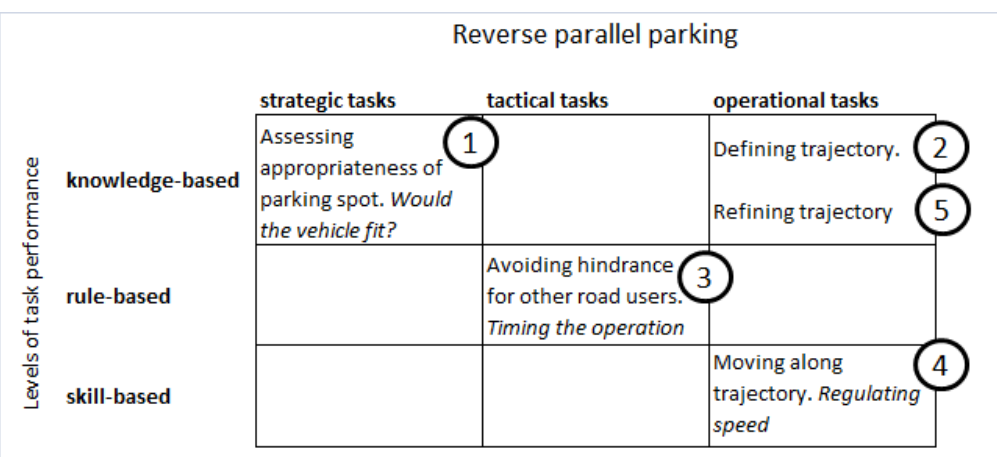

Fig. 7. Classification of subtasks for reverse parallel parking with respect to driving task-hierarchy and performance level.

Next, figure 8 visualises a comparison of the SAPP system with the preferred supported driver model. The SAPP system automatically scans for parking slots with an appropriate size. This information is available for the user upon request. In this manner the SAPP system changes the attention requiring knowledge-based task of recognising an appropriate parking spot into a skill-based task of requesting information. Furthermore, the 'knowledge' part, which involves estimating and continuously assessing the ideal trajectory, is taken over by the SAPP-system. Automation of this subtask is in line with the preferred support model, because choosing the appropriate trajectory is a demanding (knowledge-based) operational task.

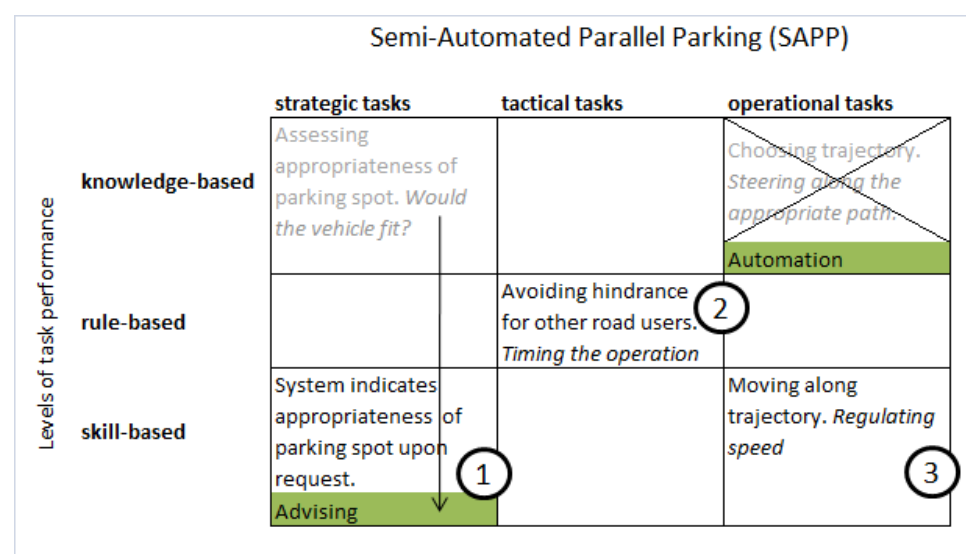

Fig. 8. Supported subtasks for reverse parallel parking when applying SAPP. 
In general, SAPP helps to change reverse parallel parking from a rather knowledge-based driving task to rule- and skill-based subtasks. The human's subtask involves supervision which he can perform at a rule-based level, the rule applies not to hinder other traffic and by breaking the driver can temporarily stop the manoeuvring, allowing vehicles to pass, before continuing again. Furthermore, automation of the trajectory reduces the amount of parallel subtask the driver is involved in. Therefore the driver can be more concentrated on the subtasks he is responsible for. One might put forth that automation of choosing trajectory reduces parking skills. However, as long as this subtask is being automated under all circumstances, this is of no matter.

\subsection{Assessment of Adaptive Cruise Control (ACC) with respect to preferred support types}

ACC supports two subtasks: remaining speed and keeping distance. To assess this assistance function with respect to the preferred support types, it is again important to review how both subtasks relate to the task-level table assuming that these tasks are executed by a person with average driving experience. See figure 9.

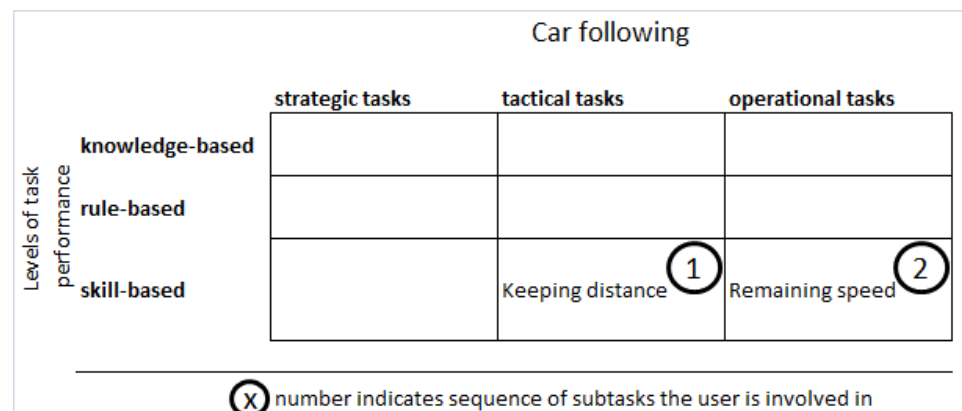

Fig. 9. Classification of subtasks for car following with respect to driving task-hierarchy and performance level.

For an average driver, the subtask to remain speed is an operational task executed at skill-based level. The classification of 'keeping distance' within the model is less obvious. It depends on traffic circumstances whether keeping distance is rewarded a tactical or operational task. Under calm traffic conditions and within familiar areas, keeping distance is an operational task. However under circumstances with heavy traffic, keeping distance is a tactical driving task. Also experienced drivers need to consider keeping distances which are long enough to avoid collision, but short enough to avoid other drivers continuously merging in front. It is within such densed traffic however for which Adaptive Cruise Control offers an advantage over normal cruise control. Therefore, keeping distance will be considered a tactical task within this research. Experienced drivers execute this task with skill-based performance.

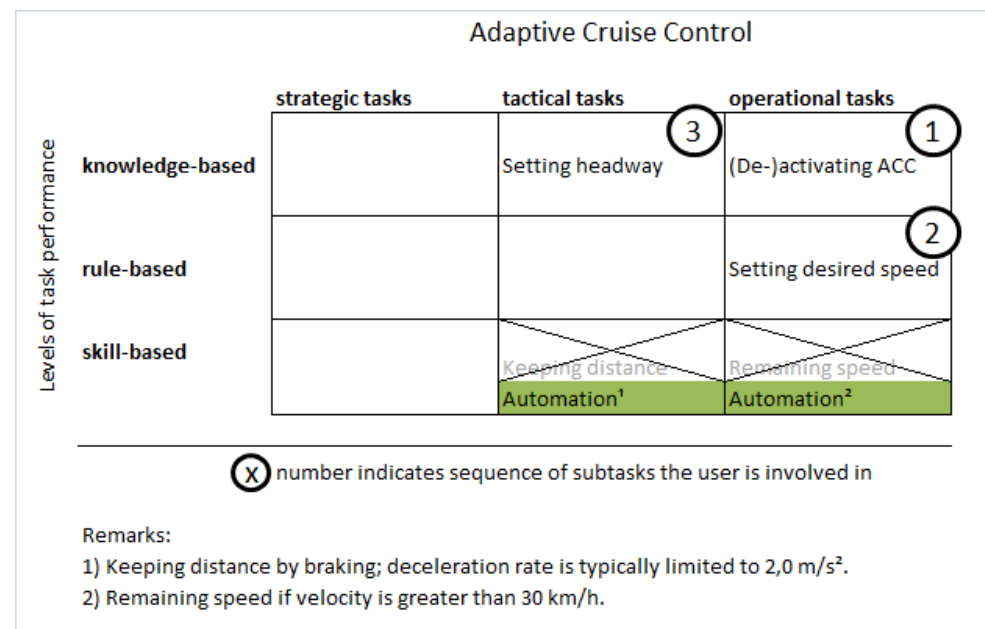

Fig. 10. Supported subtasks for cruising when applying ACC.

Figure 10 visualises a comparison of the ACC system as described in section 5.2 with the preferred supported driver model. From this comparison we conclude that the ongoing subtasks 'keeping distance' and 'remaining speed' are being supported by means of automation. For the driver new subtasks are being introduced to set speed and set headway. Besides, the driver first needs to activate the system in order to start operating. Activation and deactivation of ACC can be done manually by pressing the activation switch. Also when the driver uses the brakes, the system deactivates. When the driver brakes during a reflex-like response, deactivation is sometimes not 
immediately noticed. In general, a driver needs to consider beforehand if traffic circumstances are appropriate for applying adaptive cruising, therefore the (de-)activation task (1) is considered an operational task at knowledgebased level. As described before, keeping distance is considered a tactical task. Consequently, setting headway (3) is also a tactical task. However it will be performed at knowledge-based level. The reason is that the headway-settings are represented by a graphic within the instrument cluster and relate to three fixed time based distance stages. This means that the distance to the vehicle in front changes with speed. Evaluating and refining the settings therefore require adjustments over time.

When comparing ACC with the supported driver model and considering the frequency for operating subtasks, it is concluded that the new subtasks are performed less frequent, but require higher performance levels. This is not in line with the proposed supported driver model and might explain why drivers adapt their behaviour when using ACC [15]. Furthermore, it has to be noticed that keeping distance is not being automated under all circumstances. The driver is required to intervene by braking when a deceleration rate greater than $2,0 \mathrm{~m} / \mathrm{s}^{2}$ is necessary. Because the driver is then suddenly placed in this tactical task, this could be a possible threat. Therefore, ACC reduces the driver's subtasks under system-favourable circumstances, but suddenly increases the driver's workload under critical circumstances.

\subsection{Assessment of Lane Change Assist (LCA) with respect to preferred support types}

Again the corresponding driving task is divided into subtasks. For changing lanes the involved subtasks are indicated in figure 11 as follows: (1) Verifying the possibility for lane access, answering the question; "Is the lane to change to available?". If the lane is available the subtask for actually changing lane (2) and regulating speed (3) follow. As the first subtask involves judgement about the behaviour of other traffic, this is a tactical task which experienced drivers will operate at rule-based level under normal circumstances. One might argue if changing lanes (2) and regulating speed (3) are tactical, respectively operational tasks. Obviously, steering itself and operating the pedals are in common driving situations operational tasks. However, in situations of heavy traffic applying these operations might need cleverly timing, hence tactfulness. Therefore, changing lanes is considered a tactical task as well.

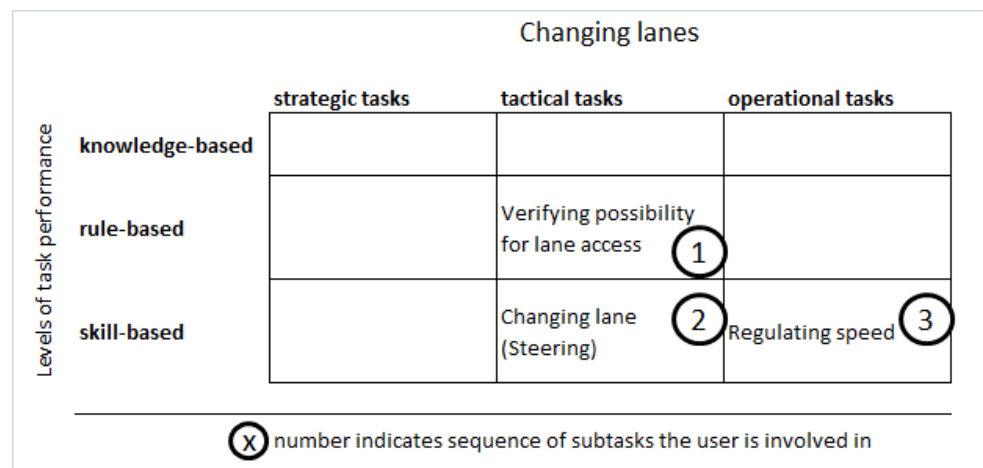

Fig. 11. Classification of subtasks for changing lanes with respect to driving task-hierarchy and performance level.

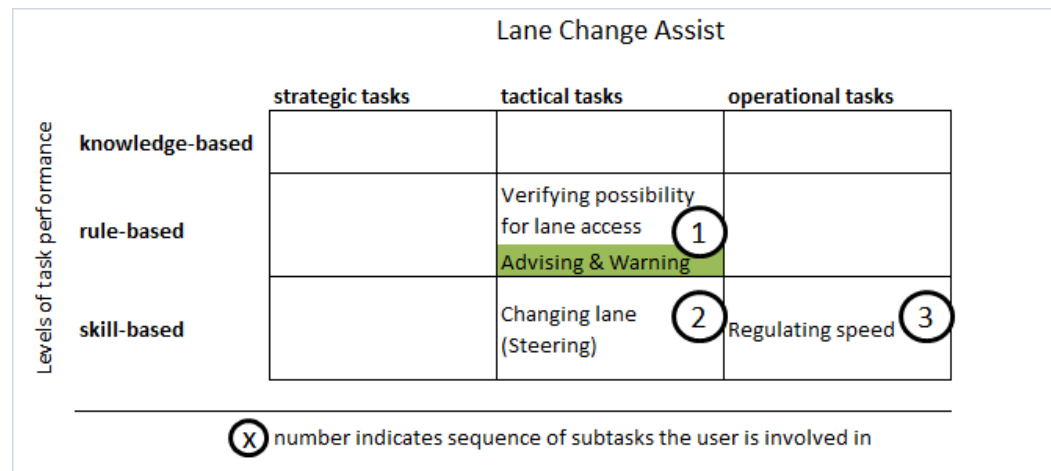

Fig. 12. Supported subtasks for changing lanes when applying LCA. 
When comparing the supported subtasks as offered by the Lane Change Assist system with the supported driver model for classification of preferred support levels, it can be concluded that LCA is in line with this model. The assistance system does not reduce the amount of involved subtasks, but among the subtasks it facilitates the most demanding one. Hence, it complies with the advise, as formulated in section 4.3, to allocate support in a manner that it enables driving tasks to be as much as possible executed on rule-based and skill-based level.

\section{Conclusions}

As mentioned in the beginning of this paper, when solutions for partly automated driving are being developed, it is important to answer in which circumstances, what type of support enhances the driver's ability to control the vehicle. Existing models describing how humans perform (driving) tasks are useful to gain insight in what subtasks can be distinguished. However, these models do not provide insight in distinguishing which subtask is in need for what kind of support. It turned out to be necessary to identify which errors can be avoided by what type of support. The performance levels distinguished within Rasmussen's model relate to typical error causes per level. This allows to recommend in general that a support system should support driving tasks to be performed at skill-based or rulebased level rather than at knowledge-based level, because drivers then operate more homogeneously and predictable.

It became clear that prerequisites for performing tasks differ per driving task's type and those prerequisites require different support. Therefore, support-types have been selected based upon their contribution in providing these prerequisites and have been combined with support-types which reduce the error causations from each different performance level (i.e. knowledge-based, rule-based and skill-based performance). This combination of allocating support in relation to performance level and driving task's type resulted in the supported-driver model and therewith relates the requested circumstances to appropriate support types. In this respect the model successfully answers the paper's initial question. It has to be noticed however, that the allocation of subtasks with respect to performance level and driver task type might sometimes be an arbitrary choice. During normal driving conditions, for example, it depends on your point of view whether lane changing is a tactical task or operational task. This ambiguity thus influences the recommended support-types, generated with the model. It needs to be noticed as well, that the model does not yet relate subtasks to timing (i.e. duration and recurrence of a task). Timing will have influence on the workload though and should therefore be related to the choice of support types too. Preferably, this aspect will be taken in consideration during further development of the model.

Nevertheless, the model has successfully been used to evaluate three existing ADAS systems with respect to the ability of the joint driver-vehicle system to remain in control under relevant circumstances. According to the model, the semi-automated parallel parking (SAPP) system, showed best allocation of support by automating the knowledge-based subtask (choosing trajectory) and adapting the remaining subtasks for the driver to rule- and skillbased level. Therewith the demanding reverse parallel parking task becomes a rather routine like operation. In this respect the SAPP system is a good example of appropriate support. As the model is successful in examining existing driving (sub)tasks with respect to possible and appropriate support types, the model is expected to be beneficial for the development of future applications of partly automated driving too. 


\section{References}

[1] European Commission, Communication on the Intelligent Car Initiative "Raising awareness of ICT for smarter, safer and cleaner vehicles", COM(2006) 59-final, European Commission, Brussels, 2002.

[2] Stevens W., Evolution to an Automated Highway System. In: P.A. loannou (Ed.), Automated Highway Systems, Plenum Press, New York, pp. 109-124, 1997.

[3] Rothengatter, T., et. al., The driver. In: J.A. Michon (Ed.), Generic Intelligent Driver Support, A comprehensive report on GIDS, Taylor \& Francis, pp. 33-52, 1993.

[4] Endsley, M., Kiris, E., The Out-of-the-loop performance problem and level of control in automation, Human Factors, 37, no. 2, pp. 381-394, 1995.

[5] Van Arem, B., et. al., The impact of Cooperative Adaptive Cruise Control on traffic-flow characteristics, IEEE Transactions on Intelligent Transportation Systems, 7, no. 4, pp. 429-436, 2006.

[6] Hollnagel, E., A function-centered approach to joint driver-vehicle system design. Cognition, Technology \& Work, 8, no. 3, pp. 169-173, 2006.

[7] McRuer, D.T., et. al., New results in driving steering control. Human Factors 19, pp. 381397, 1977.

[8] Dirken, J.M., Product-ergonomie. Ontwerpen voor gebruikers, Delft University Press, Delft, 1997.

[9] Rasmussen, J., Skills, Rules and Knowledge: Signals, Signs and other Distinctions in Human Performance Models, IEEE Transactions on Systems, Man \& Cybernatics, Vol. 13, pp. 257-266, 1983.

[10] Michon, J., A critical view of driver behavior models: what do we know, what should we do? In: Evans, L., Schwing, R.C., Human behavior and traffic safety, Plenum Press, New York, pp. 485-524, 1985.

[11] Rasmussen, J., A taxonomy for describing human malfunction in industrial installations. In: Journal of occupational accidents, 4, pp. 311-333, 1982

[12] Hale, A., et. al., Human error models as predictors of accident scenarios for designers in road transport systems, Ergonomics, 33, pp.1377-1388, 1990.

[13] Leveson, N. (2005). Adaptive Cruise Control - System Overview, Massachusetts institute of technology, Anaheim USA, 2005.

[14] Gat, I., et. al., Monocular Vision Advance Warning System for the Automotive Aftermarket, SAE World Congress \& Exhibition 2005, Detroit USA, 2005.

[15] Hoedemaker D.M., Behavioral reactions to advanced cruise control: results of a driving simulator experiment. In: Van der Heijden, R.E.C.M., Automation of Car Driving. Exploring societal impacts and conditions, Delft University Press, Delft, pp. 101-121, 2000.

Authors: $\quad$ ir. Arie Paul van den Beukel (presenting author) dr.ir. Mascha C. van der Voort

University of Twente

Laboratory Design, Production and Management PO Box 217, 7500 AE ENSCHEDE, The Netherlands

a.p.vandenbeukel@ctw.utwente.nl

m.c.vandervoort@ctw.utwente.nl

Keywords: $\quad$ Adaptive Cruise Control (ACC); Advanced Driver Assistance Systems (ADAS); automation; driver assistance; driver performance; driving task's hierarchy; LaneChange Assist (LCA); partly automated driving; Semi-automated Parallel Parking (SAPP); supported driver model; task allocation. 